\title{
Nanostructures of Ionic Liquids Confined in Pores of SBA-15: Insights from Experimental Studies and Mean-Field Density Functional Theory
}

\section{(Supporting Information)}

Dmitry N. Lapshin,,${ }^{* \dagger}$ Andrei V. Gromov, ${ }^{\ddagger}$ Eleanor E. B. Campbell, ${ }^{\ddagger}$, Lev Sarkisov"

$\dagger$ Institute for Materials and Processes, School of Engineering, University of Edinburgh, Robert Stevenson Road, Edinburgh EH9 3FB, U.K.

$\$$ EaStCHEM, School of Chemistry, University of Edinburgh, David Brewster Road, Edinburgh EH9 3FJ, U.K.

$\S$ School of Physics, Konkuk University, Seoul 05029, South Korea

|| Department of Chemical Engineering and Analytical Science, School of Engineering, University of Manchester, Manchester M13 9PL, U.K.

*Email: Dmitry.Lapshin@ed.ac.uk

The arrangement of ionic liquids (ILs) within mesoporous ordered silica SBA-15 is revealed from nitrogen physisorption experiments combined with non-local density functional theory analysis of the pore structure and mean-field density functional theory (MFDFT). Using MFDFT on simple lattice models, we outline possible scenarios for IL distributions within the pores and conditions under which they originate. MFDFT predictions for nitrogen adsorption on these models of pore structures modified with ILs are in qualitative agreement with the experimental results. They demonstrate that the distribution of the ILs inside the pores of SBA-15 is sensitive to solid-fluid interactions, leading to different structures as a function of loading. This study also recommends the MFDFT and lattice models as a powerful framework for the interpretation of nitrogen sorption behavior, which complements the existing theoretical and experimental techniques to characterize the structure of supported ILs. 


\section{Contents}

1. Mean Field Density Functional Theory $\quad$ S3

2. $\mathrm{N}_{2}$ adsorption and desorption isotherms measured at $77 \mathrm{~K} \quad \mathrm{~S} 8$

$\begin{array}{ll}3 . & \text { Pore structure characterization }\end{array}$

4. Relation between the surface area, pore volume, and radius of the cylindrical pore S12

5. MFDFT isotherms exhibiting delayed desorption and multistep condensation $\quad$ S13

6. Visualizations of fluid density states in close-ended lattice pore models $\quad$ S15 
All input files required to carry out MFDFT calculations in the canonical and grand canonical ensembles together with step-by-step instructions have been deposited in the GitHub repository https://github.com/SarkisovGroup/MFDFT.git

\section{Mean Field Density Functional Theory}

The central element of the MFDFT employed in this work is fluid and pore lattice models. In lattice models, the physical space is represented by a system of discrete lattice sites as shown in Fig. S1. Each site can be either empty or occupied by a single fluid particle. This implies that all molecule size effects and intramolecular interactions are excluded from the consideration. In the case of the confined fluid problems, some of the lattice sites are also occupied by the adsorbent particles as shown in Fig. S1. We also assume that particles interact only with the nearest-neighbour sites.

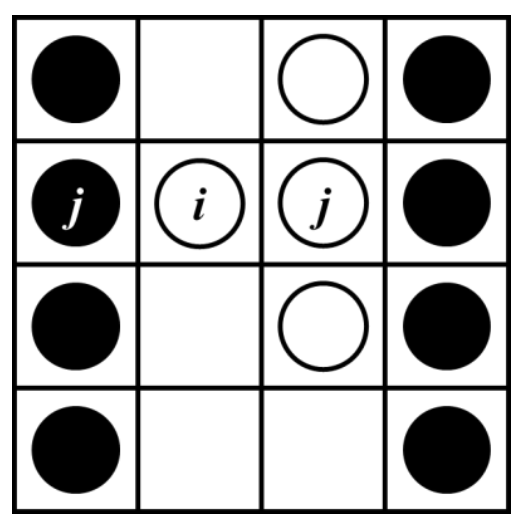

Figure S1. A lattice model of a fluid confined in a porous matrix, where black and white circles denote particles belonging to the porous matrix and fluid respectively; particles $j$ are nearest-neighbours to the particle $i$.

The Hamiltonian of a lattice gas-matrix system with only nearest-neighbour interactions can be expressed as:

$$
H=-\varepsilon_{f f} \sum \tau_{i} \tau_{j} \eta_{i} \eta_{j}-\varepsilon_{s f} \sum\left[\tau_{i} \eta_{i}\left(1-\eta_{j}\right)+\tau_{j} \eta_{j}\left(1-\eta_{i}\right)\right]
$$

where $\varepsilon_{f f}$ is the fluid-fluid energy; $\varepsilon_{s f}$ is the solid-fluid energy; $\tau$ is the occupancy of a site by fluid and is equal to 1 if the site is occupied by fluid and 0 if it is not occupied; $\eta$ is the occupancy of a site by matrix and is equal to 1 if the site is not occupied by the matrix and 0 if it is occupied. Figure $\mathrm{S} 1$ illustrates the occupancy of sites in the lattice system.

We are interested in calculating the average fluid density. Therefore, the occupancy at each fluid site in the model is expressed as an average density $\rho$ at this site and a fluctuation about the average $\delta \rho:$

$$
\tau_{i} \eta_{i}=\rho_{i}+\delta \rho_{i}=\rho_{i}+\left(\tau_{i} \eta_{i}-\rho_{i}\right)
$$

and, for two nearest-neighbour sites, we have

$$
\tau_{i} \eta_{i} \tau_{j} \eta_{j}=\left(\rho_{i}+\delta \rho_{i}\right)\left(\rho_{j}+\delta \rho_{j}\right)=\rho_{i} \rho_{j}+\rho_{j} \delta \rho_{i}+\rho_{i} \delta \rho_{j}+\delta \rho_{i} \delta \rho_{j}
$$


If we now apply the mean-field approximation, whereby the higher-order density fluctuations $\delta \rho_{i} \delta \rho_{j}$ are neglected and substitute an expression for $\delta \rho_{i}=\left(\tau_{i} \eta_{i}-\rho_{i}\right)$, from equation (S2), we obtain

$$
\tau_{i} \eta_{i} \tau_{j} \eta_{j}=-\rho_{i} \rho_{j}+\rho_{i} \tau_{j} \eta_{j}+\rho_{j} \tau_{i} \eta_{i}
$$

Thermodynamics of confined fluid in a porous material (or a rigid lattice matrix) can be described by the grand potential $(\Omega)$ :

$$
\Omega=U-T S-\mu N
$$

where $S$ is the entropy, $U$ is the energy, $T$ is the temperature, $\mu$ is the chemical potential, $N$ is the number of particles in the system.

The statistical mechanics definition for the grand potential is

$$
\Omega=-k T \ln \Xi
$$

where $k$ is the Boltzmann constant, and the grand partition function $\Xi$ within the MFT formalism is equal to

$$
\Xi=\sum_{\tau} e^{\frac{-\left(H_{m f}-\mu N\right)}{k T}}
$$

Rewriting equations (S1) by taking into consideration the mean-field approximation in equation (S4) and substituting the resulting equation for $H_{m f}$, we obtain

$$
\Xi=\sum_{\tau} \exp \left(-\beta \varepsilon_{f f} \sum_{i<j} \rho_{i} \rho_{j}\right) \cdot \sum_{\tau} \exp \left(\beta\left[\varepsilon_{f f} \sum_{j} \rho_{j}+\varepsilon_{s f} \sum_{j}\left(1-\eta_{j}\right)+\mu\right] \cdot \sum_{i} \tau_{i} \eta_{i}\right)
$$

where $\beta=1 / k T$. Here, quantities $H_{0}$ and $X_{i}$ are defined as

$$
\begin{gathered}
H_{0}=\varepsilon_{f f} \sum_{i<j} \rho_{i} \rho_{j} \\
X_{i}=\beta\left[\varepsilon_{f f} \sum_{j} \rho_{j}+\varepsilon_{s f} \sum_{j}\left(1-\eta_{j}\right)+\mu\right]
\end{gathered}
$$

Thus, equation (S8) can be rewritten as

$$
\Xi=\sum_{\tau} \exp \left(-\beta H_{0}\right) \cdot \sum_{\tau} \exp \left(X_{i} \cdot \sum_{i} \tau_{i} \eta_{i}\right)
$$


After rearrangement, the grand partition function is equal to

$$
\Xi=\sum_{\tau} \mathrm{e}^{-\beta H_{0}} \cdot \sum_{\tau} \prod_{i} e^{X_{i} \tau_{i}}
$$

In equation (S12) the summation over $\tau$ denotes the summation not over single occupancy values 0 or 1 but over all possible permutations of 0 and 1 and denoted as $\{\tau\}$. Then, we can write

$$
\sum_{\{\tau\}} \prod_{i} e^{X_{i} \tau_{i}}=\prod_{i} \sum_{\{\tau\}} e^{X_{i} \tau_{i}}=\prod_{i}\left(1+e^{X_{i}}\right)
$$

In equation (S13) the product goes only over $(i) \mathrm{s}$ which are not occupied by matrix, because for sites occupied by matrix $\tau_{i}=0$ and the first product in equation (S13) reduces to 1 . Therefore, in our further derivations, a property or a sum labelled $(i)$ is considered to be a property or a sum for all sites $i$ not occupied by matrix.

The grand partition function becomes

$$
\Xi=\mathrm{e}^{-\beta H_{0}} \cdot \prod_{i}\left(1+e^{X_{i}}\right)
$$

Substituting equation (S14) into equation (S6), we obtain the following expression for the grand potential functional

$$
\Omega\left[\rho_{i}\right]=-k T\left[-\beta H_{0}+\sum_{i} \ln \left(1+e^{X_{i}}\right)\right]
$$

where the notation $\left[\rho_{i}\right]$ stands for the set of densities for all the lattice sites in the system. For clarity, we omit this notation in our further derivations and use it where necessary.

The mean density at site $i$ is determined as a partial derivative of the grand potential with respect to the chemical potential

$$
\sum_{i} \rho_{i}=-\left(\frac{\partial \Omega}{\partial \mu}\right)_{V, T}=\frac{1}{\beta} \cdot \frac{\partial \ln (\Xi)}{\partial \mu}=\sum_{i} \frac{1}{\left(1+e^{-X_{i}}\right)}
$$

where $\sum_{i} \rho_{i}=N$, and $V$ is the volume of the system.

Therefore, the equation for average fluid density at site $i$ is equal to

$$
\rho_{i}=\frac{1}{\left(1+e^{-X_{i}}\right)}
$$


Equivalently, equation (S17) can be obtained after defining the external field at site $i$ according to equation (S18)

$$
\varphi_{i}=-\varepsilon_{s f} \sum_{j}\left(1-\eta_{j}\right)
$$

And, then, similar to equation (S16)

$$
\sum_{i} \rho_{i}=-\left(\frac{\partial \Omega}{\partial\left(\mu-\varphi_{i}\right)}\right)_{V, T}=\sum_{i} \frac{1}{\left(1+e^{-X_{i}}\right)}
$$

The solution (S17) satisfies the necessary equilibrium condition (S20)

$$
\left(\frac{\partial \beta \Omega}{\partial \rho_{i}}\right)_{\mu, V, T}=0
$$

Equation (S17) indicates that for $N$ sites there are $N$ non-linear equations. In this work, we solved these equations by iteration.

Finally, we define the mean-field approximation of the Helmholtz free energy for the lattice system according to the equation

$$
F\left(\left[\rho_{i}\right]\right)=\Omega\left(\left[\rho_{i}\right]\right)+\mu \sum_{i}\left(\rho_{i}\right)
$$

or, if the external field is present

$$
F\left(\left[\rho_{i}\right]\right)=\Omega\left(\left[\rho_{i}\right]\right)-\left(\varphi_{i}-\mu\right) \sum_{i}\left(\rho_{i}\right)
$$

The next three expressions define the necessary components of equations (S21) and (S22)

$$
\begin{gathered}
\Omega=-\frac{1}{\beta}\left[-\beta H_{0}+\sum_{i} \ln \left(1+e^{X_{i}}\right)\right] \\
e^{X_{i}}=\frac{\rho_{i}}{1-\rho_{i}} \\
\left(\varphi_{i}-\mu\right)=\varepsilon_{f f} \sum_{j} \rho_{j}-\frac{X_{i}}{\beta}
\end{gathered}
$$

We now substitute equations (S23) into equation (S21) and obtain

$$
F=-\frac{\varepsilon_{f f}}{2} \sum_{i<j} \rho_{i} \rho_{j}+\sum_{i}\left[\rho_{i} \ln \left(\rho_{i}\right)+\left(1-\rho_{i}\right) \ln \left(1-\rho_{i}\right)\right]+\sum_{i} \rho_{i} \varphi_{i}
$$


The necessary condition for the Helmholtz free energy minimum in the canonical ensemble is:

$$
\left(\frac{\partial F}{\partial \rho_{i}}\right)_{N, V, T}-\mu=0
$$

which gives an expression for the chemical potential at site $i$, which can also be directly written from equation (S10):

$$
\mu_{i}=k T \ln \left(\frac{\rho_{i}}{\left(1-\rho_{i}\right)}\right)-\varepsilon_{f f} \sum_{j} \rho_{j}+\varphi_{i}
$$

This indicates that the solution for the minimum of the grand potential and the Helmholtz free energy is the same and is given by equation (S17), which is solved iteratively for fluid density with different imposed constraints. In the grand ensemble, equation (S17) is solved at fixed uniform chemical potential $\mu_{i}=\mu$. In the canonical ensemble, the equation is solved with the constraint of the constant overall density $\sum_{i} \rho_{i}=N$. 
2. $\mathrm{N}_{2}$ adsorption and desorption isotherms measured at $77 \mathrm{~K}$

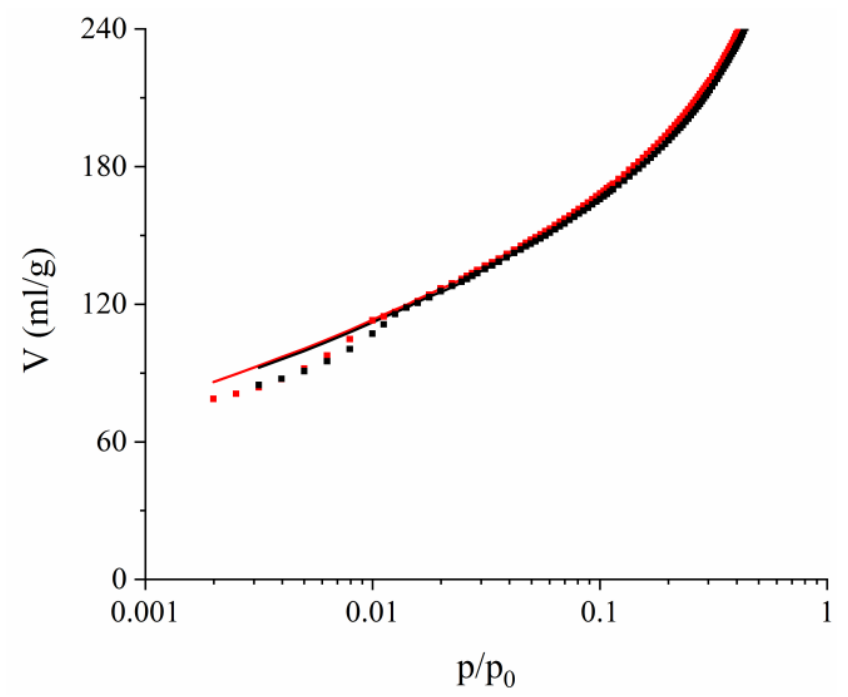

(a)

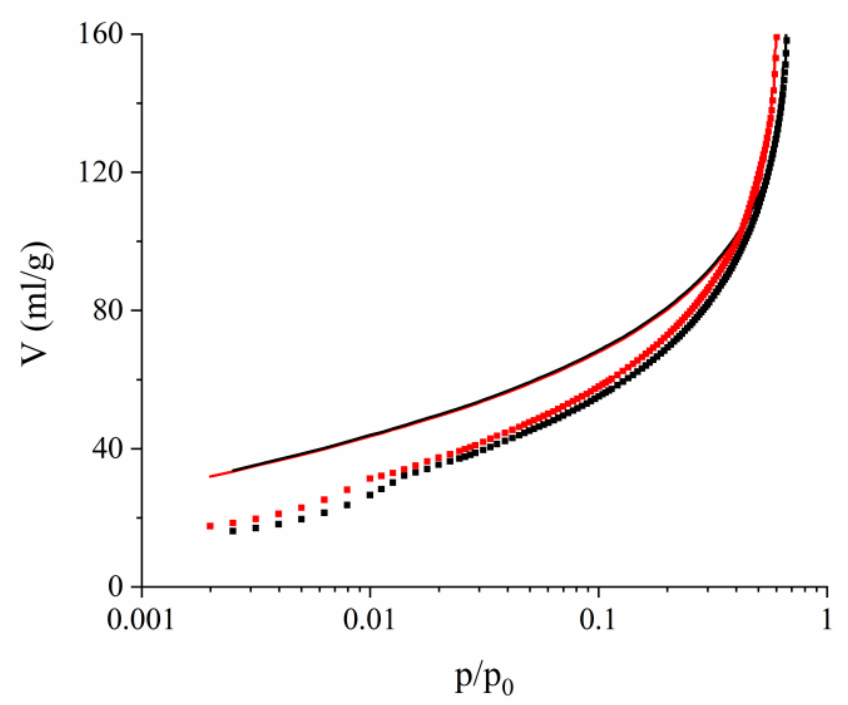

(c)

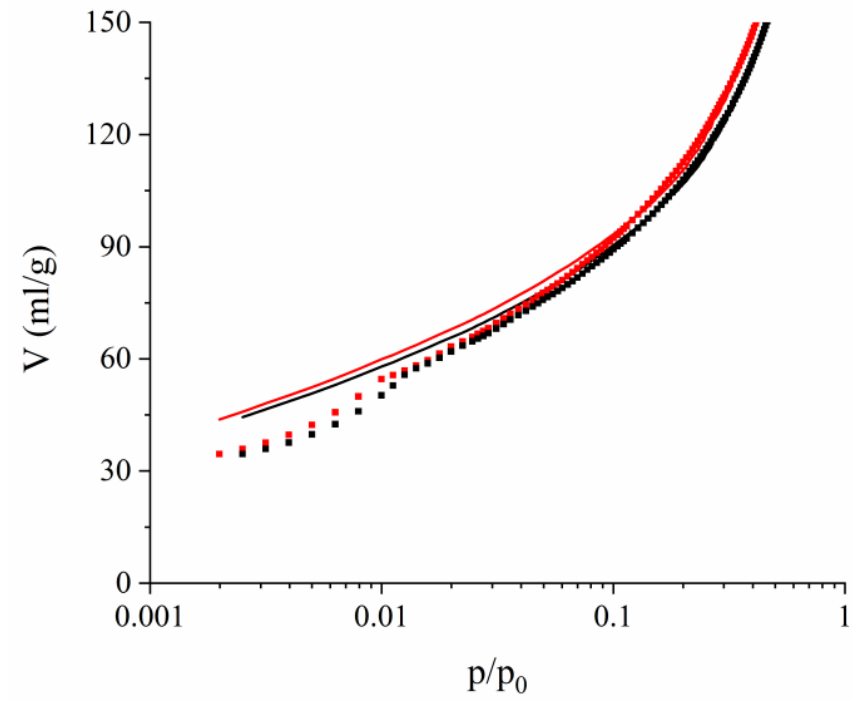

(b)

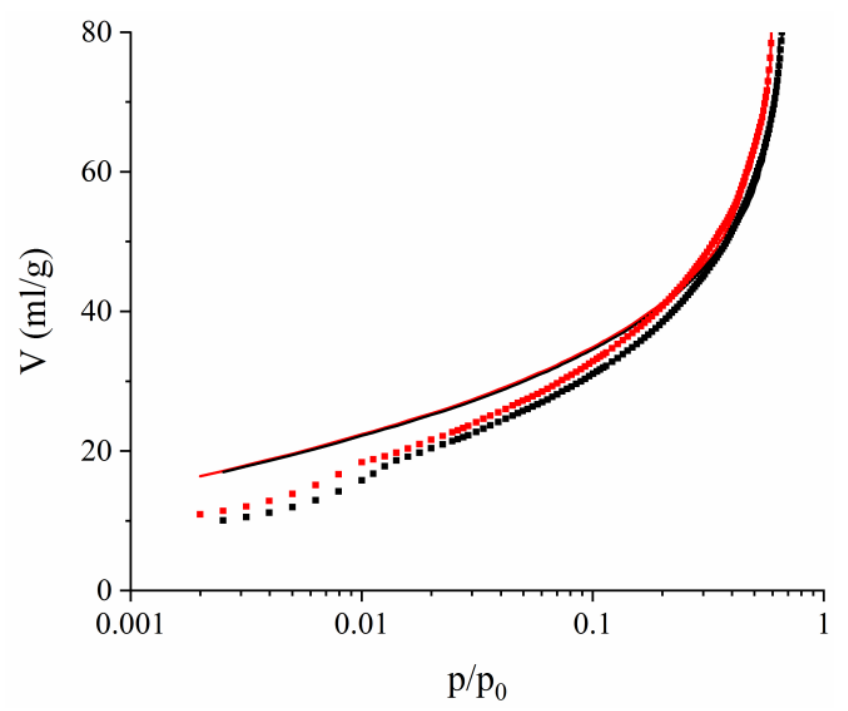

(d)

Figure S2. Nitrogen adsorption and desorption isotherms measured at $77 \mathrm{~K}$ for SBA-15 impregnated with different amounts of IL $\left[\mathrm{C}_{4} \mathrm{C}_{1} \mathrm{Im}\right]^{+}\left[\mathrm{PF}_{6}\right]^{-}$corresponding to the following pore filling degrees: (a) $0.0 \%$; (b) $12.8 \%$; (c) $20.6 \%$; (d) $48.5 \%$. Dots are the experimental results; lines are the fitted isotherms obtained from the NLDFT with a kernel for silica cylindrical pore shape; black and red colors correspond to adsorption and desorption branches of the isotherm. 


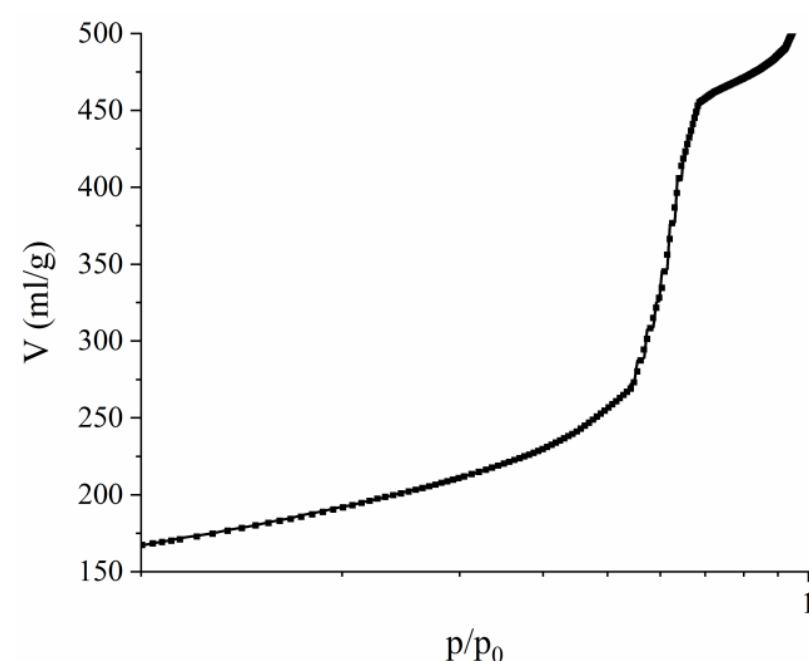

(a)

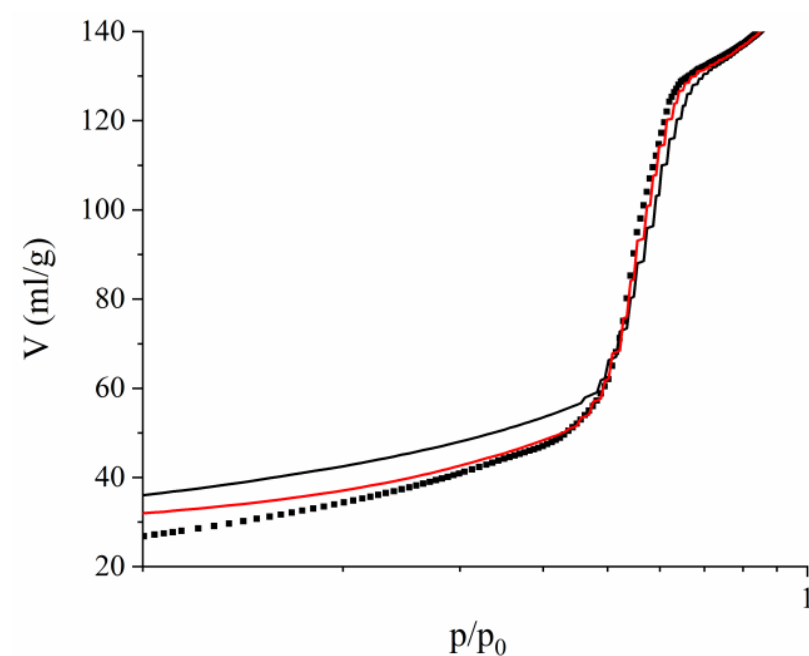

(d)

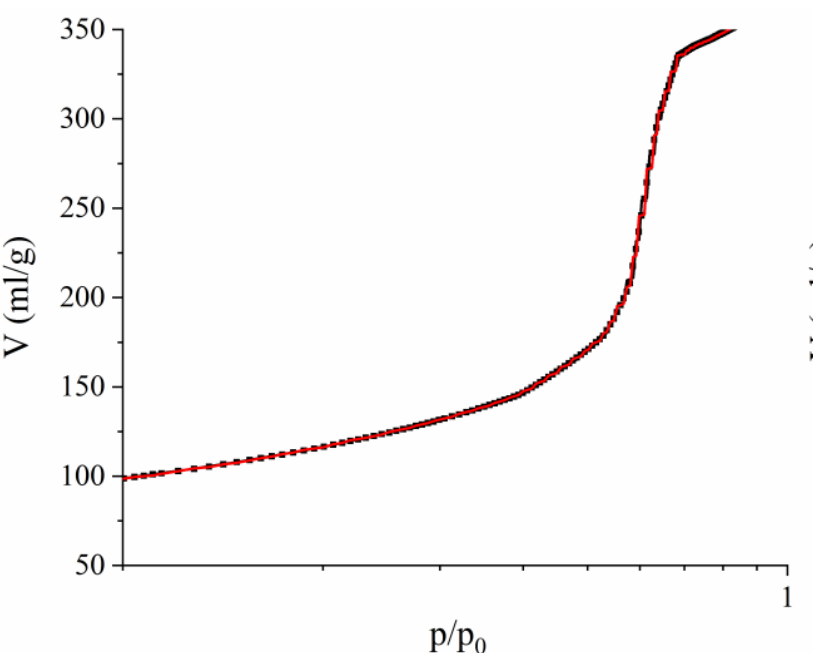

(b)

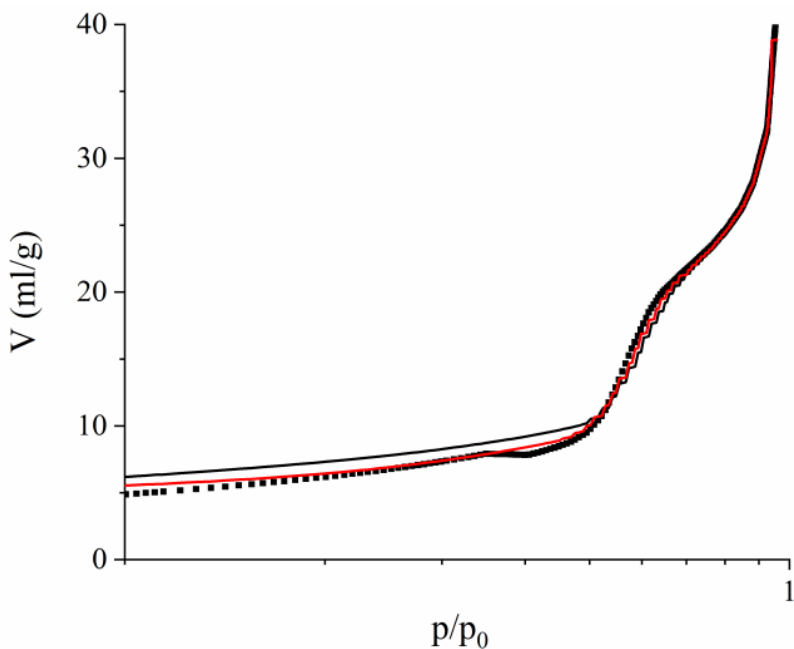

(e)

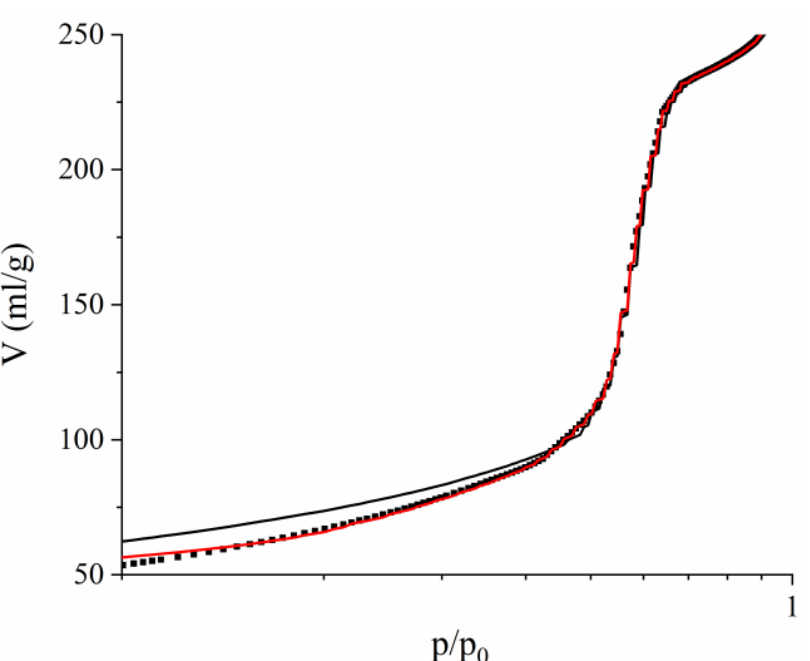

(c)

Figure S3. Nitrogen desorption isotherms measured at $77 \mathrm{~K}$ for SBA-15 impregnated with different amounts of $\mathrm{IL}_{[}\left[\mathrm{C}_{2} \mathrm{C}_{1} \mathrm{Im}\right]^{+}\left[\mathrm{Tf}_{2} \mathrm{~N}\right]^{-}$corresponding to the following pore filling degrees: (a) $0.0 \%$; (b) $11.0 \%$; (c) $31.2 \%$; (d) $54.5 \%$; (e) $91.7 \%$. Dots are the experimental results; lines are the fitted isotherms obtained from the NLDFT with a kernel for silica (black) and carbon (red) cylindrical pore shape. 


\section{Pore structure characterization}

Figure S4 illustrates PSDs obtained from the adsorption and desorption branches of the $\mathrm{N}_{2}$ isotherm measured for samples of SBA-15 impregnated with $\left[\mathrm{C}_{4} \mathrm{C}_{1} \mathrm{Im}\right]^{+}\left[\mathrm{PF}_{6}\right]^{-}$. Two PSDs exhibit similar shapes indicating the absence of pore-blocking effects due to the presence of the confined IL.

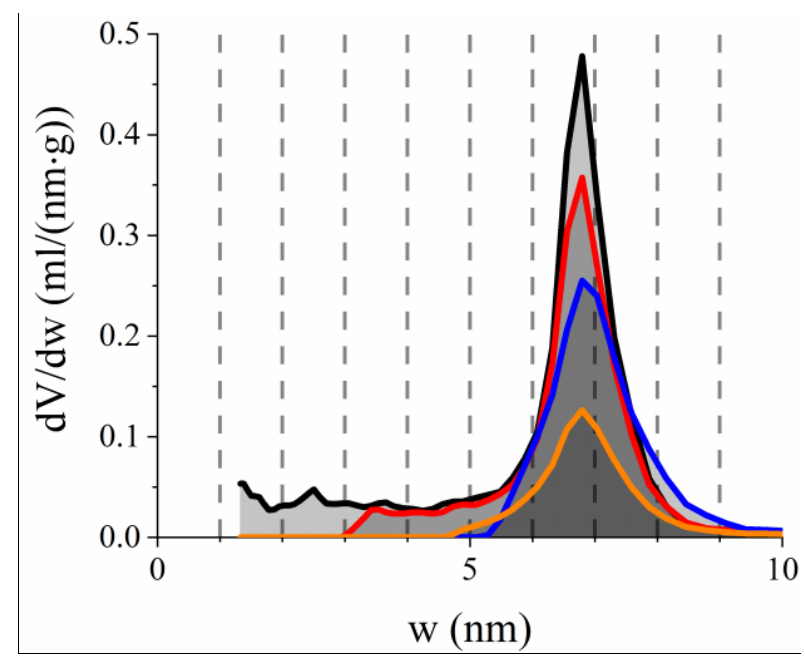

(a)

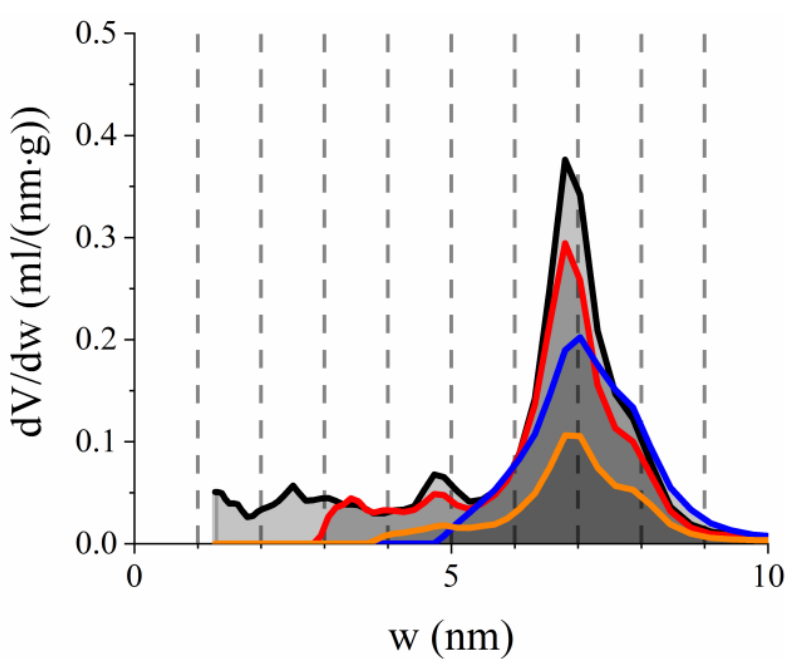

(b)

Figure S4. NLDFT pore size distributions obtained from the adsorption (a) and desorption (b) branches of the isotherm of pure SBA-15 (black), and filled with the $\mathrm{IL}\left[\mathrm{C}_{4} \mathrm{C}_{1} \mathrm{Im}\right]^{+}\left[\mathrm{PF}_{6}\right]^{-}$for the following pore filling degrees (a) 14,4\% (red), $23.2 \%$ (blue), $54.5 \%$ (orange); (b) 12,8\% (red), $20.6 \%$ (blue), 48.5 $\%$ (orange). 
Table S1 presents results that were obtained from the analysis of isotherms and the application of NLDFT kernels with the best fit to the experimental adsorption and desorption branches of isotherms. For $\left[\mathrm{C}_{4} \mathrm{C}_{1} \mathrm{Im}\right]^{+}\left[\mathrm{PF}_{6}\right]^{-}$we compared the results obtained from adsorption and desorption branches using a $\mathrm{SiO}_{2}$-kernel. In contrast, for $\left[\mathrm{C}_{2} \mathrm{C}_{1} \mathrm{Im}\right]^{+}\left[\mathrm{Tf}_{2} \mathrm{~N}\right]^{-}$we decided to compare $\mathrm{SiO}_{2}$ and C-kernels for the equilibrium isotherms that work best to reflect morphological and chemical composition changes observed in mesopores upon impregnation of the IL.

Table S1. Pore structure characterization of SBA-15 impregnated with $\left[\mathrm{C}_{4} \mathrm{C}_{1} \mathrm{Im}\right]^{+}\left[\mathrm{PF}_{6}\right]^{-}$or $\left[\mathrm{C}_{2} \mathrm{C}_{1} \mathrm{Im}\right]^{+}\left[\mathrm{Tf}_{2} \mathrm{~N}\right]^{-}$.

\begin{tabular}{|c|c|c|c|c|c|c|c|c|c|c|c|c|c|c|c|c|}
\hline \# & $\begin{array}{l}\text { Compo } \\
\text { SBA-15 }\end{array}$ & $\begin{array}{l}\text { ition, } \\
\text { IL }\end{array}$ & $\begin{array}{c}V_{I L} \\
\mathrm{ml} / \mathrm{g}\end{array}$ & $\begin{array}{l}S_{\text {total }} \\
\mathrm{m}^{2} / \mathrm{g}\end{array}$ & $\begin{array}{c}\Delta S_{1} \\
m^{2} / g\end{array}$ & $\begin{array}{l}\Delta S_{2} \\
m^{2} / g\end{array}$ & $\begin{array}{c}\Delta S_{2} / \Delta S_{1} \\
\%\end{array}$ & $\begin{array}{l}V_{\text {total }} \\
\mathrm{ml} / \mathrm{g}\end{array}$ & $\begin{array}{l}\Delta V_{l} \\
\mathrm{ml} / \mathrm{g}\end{array}$ & $\begin{array}{l}\Delta V_{2} \\
\mathrm{ml} / \mathrm{g}\end{array}$ & $\begin{array}{c}\Delta V_{2} / \Delta V_{1} \\
\%\end{array}$ & $\begin{array}{l}V_{\text {free }} \\
\mathrm{ml} / \mathrm{g}\end{array}$ & $\begin{array}{c}\Delta V_{\text {iwall }} \\
\mathrm{ml} / \mathrm{g}\end{array}$ & $\begin{array}{c}\Delta V_{\text {iwall }}+\left(\Delta V_{l}-\Delta V_{2}\right) \\
\mathrm{ml} / \mathrm{g}\end{array}$ & $\begin{array}{c}V_{\text {block }} \\
\%\end{array}$ & $\begin{array}{l}\delta \\
\%\end{array}$ \\
\hline \multicolumn{17}{|c|}{$\left[\mathrm{C}_{4} \mathrm{C}_{1} \mathrm{Im}^{+}\left[\mathrm{PF}_{6}\right]^{-}\right.$} \\
\hline $1 \mathrm{D}$ & 1.000 & 0.000 & - & 653 & - & - & - & 0.725 & - & - & - & 0.725 & 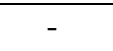 & - & 0,0 & 0.0 \\
\hline $1 \mathrm{~A}$ & 1.000 & 0.000 & - & 644 & - & - & - & 0.714 & - & - & - & 0.714 & - & - & 0.0 & 0.0 \\
\hline $2 \mathrm{D}$ & 0.877 & 0.123 & 0.090 & 326 & 216 & 202 & 94 & 0.523 & 0.400 & 0.375 & 94 & 0.546 & 0.088 & 0.113 & 3.55 & 12.8 \\
\hline $2 \mathrm{~A}$ & 0.877 & 0.123 & 0.090 & 313 & 241 & 229 & 95 & 0.514 & 0.426 & 0.412 & 97 & 0.537 & 0.101 & 0.115 & 3.56 & 14.4 \\
\hline $3 \mathrm{D}$ & 0.816 & 0.185 & 0.135 & 240 & 204 & 210 & 103 & 0.437 & 0.369 & 0.390 & 106 & 0.456 & 0.174 & 0.153 & 3.15 & 20.6 \\
\hline $3 \mathrm{~A}$ & 0.816 & 0.185 & 0.135 & 236 & 222 & 211 & 95 & 0.426 & 0.389 & 0.375 & 96 & 0.447 & 0.140 & 0.154 & 3.66 & 23.2 \\
\hline $4 \mathrm{D}$ & 0.653 & 0.347 & 0.254 & 121 & 161 & 84 & 52 & 0.212 & 0.291 & 0.156 & 54 & 0.219 & 0.128 & 0.263 & 1.47 & 48.5 \\
\hline $4 \mathrm{~A}$ & 0.653 & 0.347 & 0.254 & 117 & 176 & 97 & 55 & 0.207 & 0.318 & 0.173 & 54 & 0.212 & 0.114 & 0.259 & 1.21 & 54.5 \\
\hline \multicolumn{17}{|c|}{$\left[\mathrm{C}_{2} \mathrm{C}_{1} \mathrm{Im}\right]^{+}\left[\mathrm{Tf}_{2} \mathrm{~N}\right]^{-}$} \\
\hline $5 \mathrm{SiO}_{2}$ & 1.000 & 0.000 & - & 624 & - & - & & 0.710 & - & - & - & 0.710 & - & - & 0.0 & 0.0 \\
\hline $6 \mathrm{SiO}_{2}$ & 0.894 & 0.106 & 0.070 & 352 & 198 & 224 & 113 & 0.526 & 0.396 & 0.395 & 100 & 0.565 & 0.109 & 0.110 & 6.18 & 11.0 \\
\hline $6 \mathrm{C}$ & 0.894 & 0.106 & 0.070 & 406 & 198 & 191 & 96 & 0.534 & 0.396 & 0.363 & 92 & 0.565 & 0.066 & 0.099 & 4.93 & 11.0 \\
\hline $7 \mathrm{SiO}_{2}$ & 0.748 & 0.252 & 0.166 & 220 & 167 & 206 & 123 & 0.363 & 0.331 & 0.334 & 101 & 0.366 & 0.171 & 0.171 & 0.52 & 31.2 \\
\hline $7 \mathrm{C}$ & 0.748 & 0.252 & 0.166 & 228 & 167 & 168 & 101 & 0.369 & 0.331 & 0.289 & 87 & 0.366 & 0.119 & 0.161 & -0.62 & 31.2 \\
\hline $8 \mathrm{SiO}_{2}$ & 0.630 & 0.370 & 0.244 & 126 & 139 & 121 & 87 & 0.206 & 0.278 & 0.191 & 69 & 0.203 & 0.155 & 0.242 & -0.59 & 54.5 \\
\hline $8 \mathrm{C}$ & 0.630 & 0.370 & 0.244 & 128 & 139 & 124 & 89 & 0.210 & 0.278 & 0.197 & 71 & 0.203 & 0.155 & 0.236 & -1.38 & 54.5 \\
\hline $9 \mathrm{SiO}_{2}$ & 0.503 & 0.497 & 0.328 & 19 & 112 & 17 & 15 & 0.036 & 0.222 & 0.027 & 12 & 0.030 & 0.125 & 0.319 & -1.74 & 91.7 \\
\hline $9 \mathrm{C}$ & 0.503 & 0.497 & 0.328 & 20 & 112 & 17 & 15 & 0.036 & 0.222 & 0.030 & 13.5 & 0.030 & 0.125 & 0.317 & -1.85 & 91.7 \\
\hline
\end{tabular}

A and D - the results are calculated from the adsorption (A) and desorption (D) isotherms obtained from the NLDFT with a kernel for silica cylindrical pore shape. $\mathrm{SiO}_{2}$ and $\mathrm{C}$ - the results are calculated from the desorption isotherms obtained from the NLDFT with a kernel for silica and carbon cylindrical pore shape. 


\section{Relation between the surface area, pore volume, and radius of the cylindrical pore}

The thickness of the IL layer can be calculated assuming that an SBA-15 material consists of cylindrical pores with a radius $R$, length $h$, surface area $S$, and total pore volume $V$. The surface area and volume of empty cylindrical pores is equal to

$$
\begin{aligned}
& S=2 \pi R h \\
& V=\pi R^{2} h
\end{aligned}
$$

We assume that the confined IL uniformly spreads over the surface. The adsorbed layer of the IL reduces the radius, surface area, and total pore volume of the pure SBA-15 to corresponding values $r, s$, and $v$ respectively

$$
\begin{aligned}
& s=2 \pi r h \\
& v=\pi r^{2} h
\end{aligned}
$$

Using the geometrical parameters of the cylindrical pore (Fig. S5) we can calculate the volume of the IL covering the pore surface as

$$
\Delta V=V-v=\pi R^{2} h-\pi r^{2} h=\pi h\left(R^{2}-r^{2}\right)=\pi h(R-r)(R+r)
$$

Finally, we can find the thickness of the IL layer as the radii difference $(R-r)$

$$
(R-r)=\frac{\Delta V}{\pi h(R+r)}=\frac{2 \Delta V}{S+S}
$$

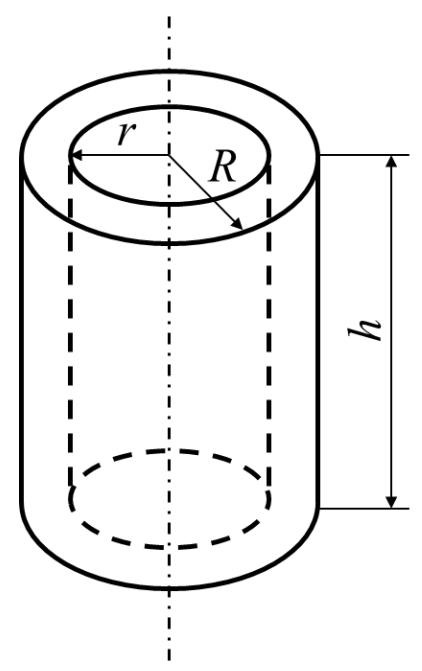

Figure S5. Schematic illustration of a cylindrical pore with the radius $R$, length $h$. A smaller cylinder with the radius $r$ represents a pore with a uniformly adsorbed IL layer with the thickness $R-r$. 


\section{MFDFT isotherms exhibiting delayed desorption and multistep condensation}

Figure S6 illustrates how the presence of intrawall pores wider than 5 lattice sites influence the shape of $\mathrm{N}_{2}$ isotherms for the slit pore containing some amount of pre-adsorbed IL. These isotherms have been calculated using MFDFT.

The red isotherm in Fig. S6(a) is calculated for the pore covered with a monolayer of IL ( $\alpha=$ 1.0) as shown in the visualization below. At IL loading equal to 0.314 the mean size of the main mesopore is reduced from 8 to 6 lattice sites in comparison to the empty pore (black curve). Moreover, intrawall pores with the width of 6 lattice sites are covered with a monolayer while the smaller ones are completely filled with the IL. In some regions of the pore, IL forms narrow necks that lead to the appearance of ink-bottle slit pores. A simple schematics in Fig. S6(b) shows how ink-bottle pores form as IL adsorbs on the surface (steps 1 and 2). Desorption of nitrogen (step 4) in this pore is delayed as illustrated by the red isotherm in Fig. S6(a) due to the cavitation effect when a bubble grows in the pore with a decrease of partial pressure (relative activity). Once the relative activity reaches a value of 0.65 the main mesopore empties through intrawall pores.
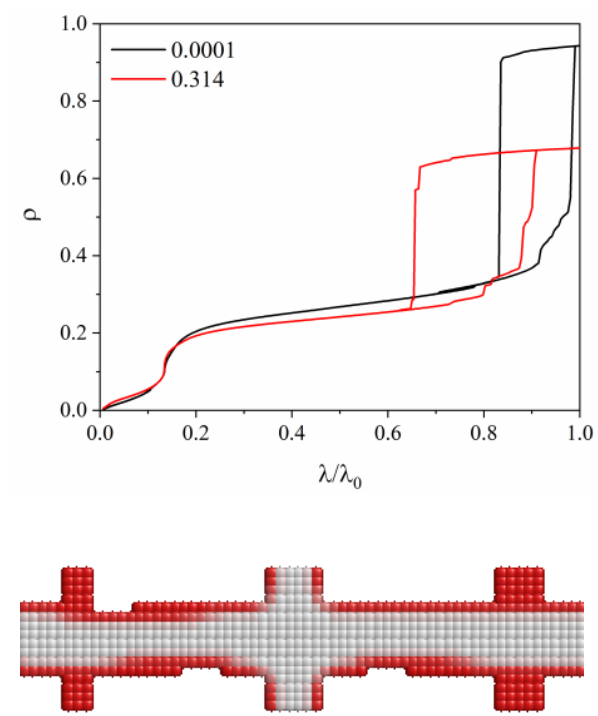

(a)

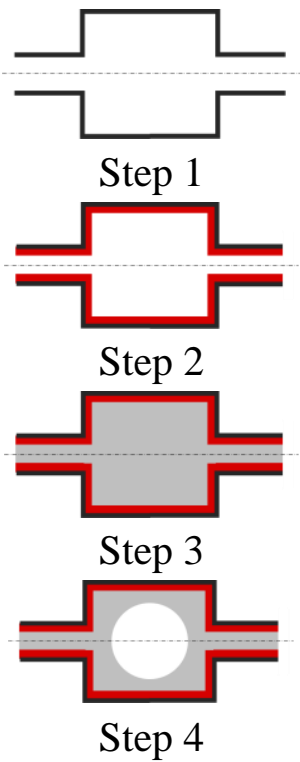

(b)
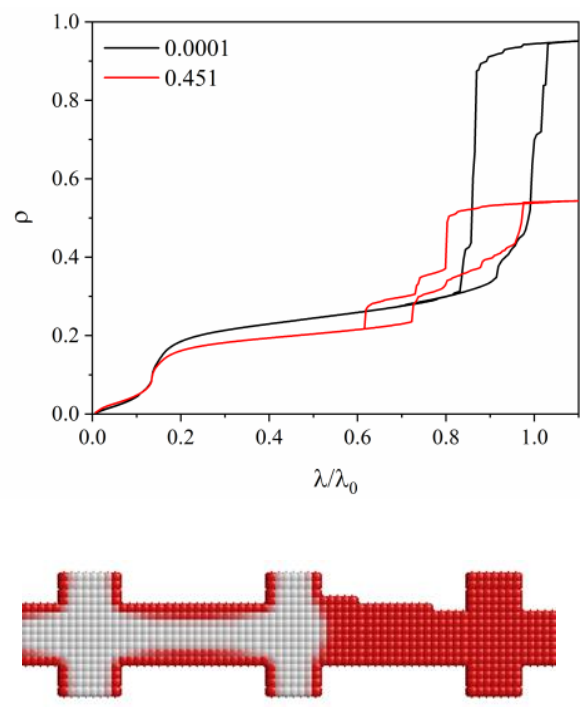

(c)

Figure S6. Density isotherms or average fractional occupancy for the lattice model of $\mathrm{N}_{2}$ in empty slit pores (black curves) and pores containing pre-adsorbed IL at some IL loading (red curves). The mean width of the main mesopore is equal to 8 lattice sites and the widths of intrawall pores are uniformly distributed from 2 to 6 lattice sites for (a) and from 2 to 8 lattice sites for (c). The strength of solid-IL interaction $\alpha=1.0$ for (a) and 2.0 for (c). Visualizations show the most representative sections of the slit pore channel to illustrate the behavior of IL (red sites). (b) A schematics of an ink-bottle slit pore: step 1 - the empty pore; step 2 - the pore with a monolayer of IL (red area); step 3 - the pore containing the IL with nitrogen condensed in it; step 4 - formation of a bubble due to the cavitation effect upon desorption of nitrogen from the pore.

Figure S6(c) shows $\mathrm{N}_{2}$ isotherms calculated for the pore network consisting of the main mesopore with the mean size of 8 lattice sites and intrawall pores ranging in size from 2 to 8 lattice 
sites. At IL loading equal to 0.451 and $\alpha=2.0$, the liquid completely covers the surface and also forms multiple liquid bridges as shown in Fig. S6(c). From the visualization, we also notice that intrawall pores with the size 7 and 8 lattice sites are not completely filled with the IL. The red isotherm shows multistep condensation and desorption where each step is associated with the corresponding process in pores of different diameters. 


\section{Visualizations of fluid density states in close-ended lattice pore models}

Figure $\mathrm{S} 7$ visualizes density states of $\mathrm{N}_{2}$ adsorbing in or desorbing from close-ended lattice pore models without (a) and with (b) a pore narrowing. According to Fig. S7(a) adsorption of $\mathrm{N}_{2}$ in a simple close-ended pore proceeds through gradual filling of the free pore space. Similarly, desorption (not shown) occurs through gradual emptying of the pore. Adding a single pore narrowing as illustrated in Fig. S7(b) does not affect the adsorption process. However, it changes the desorption of the fluid from the pore. First, the fluid gradually desorbs from the pore until it reaches the narrowing. This narrowing blocks further desorption leading to a shift of the equilibrium pressure to a lower value at which desorption occurs for a pore with a width of 6 sites. Once a new pressure is reached the pore empties leaving a monolayer of the adsorbed fluid. At the lowest pressure, the pore completely empties.
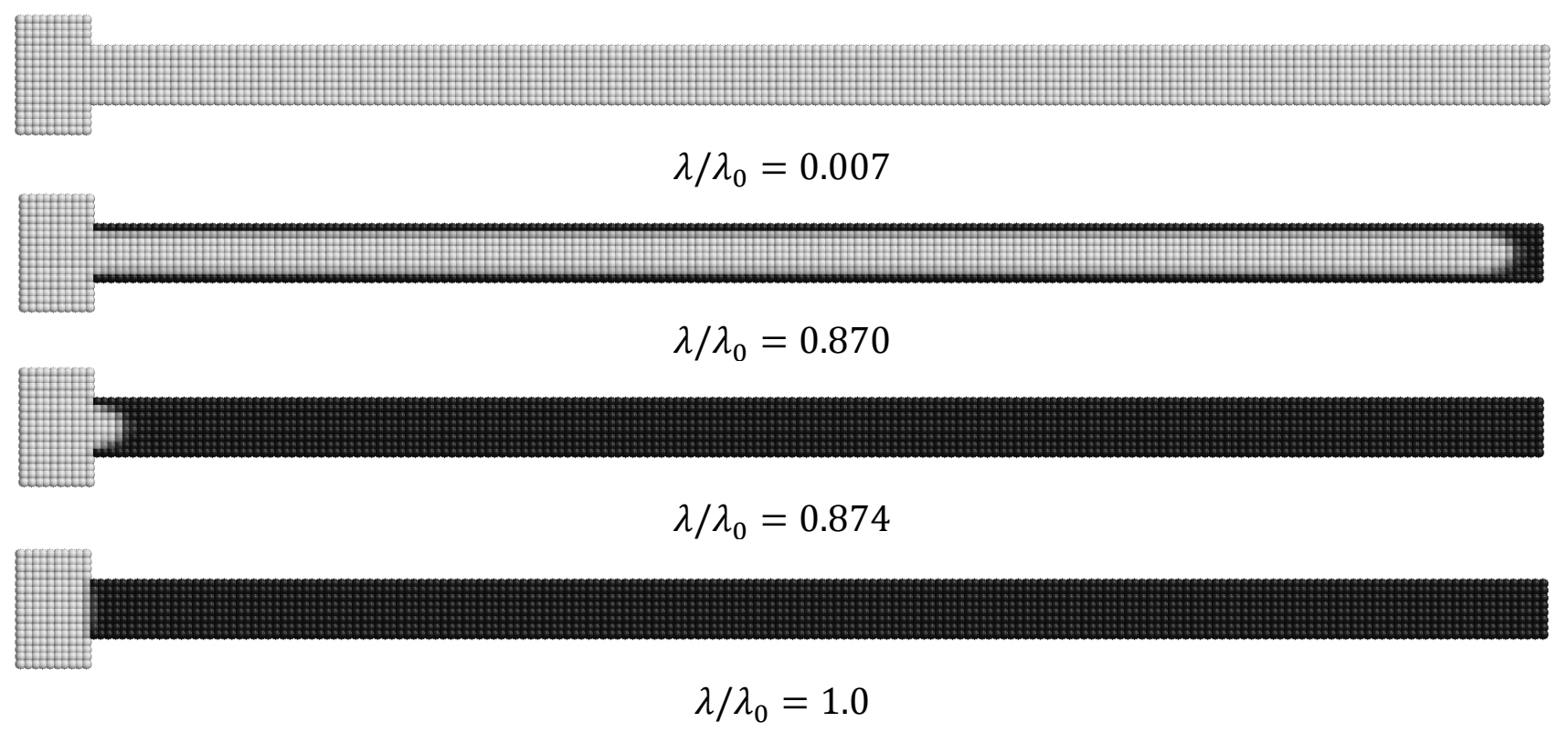

(a)
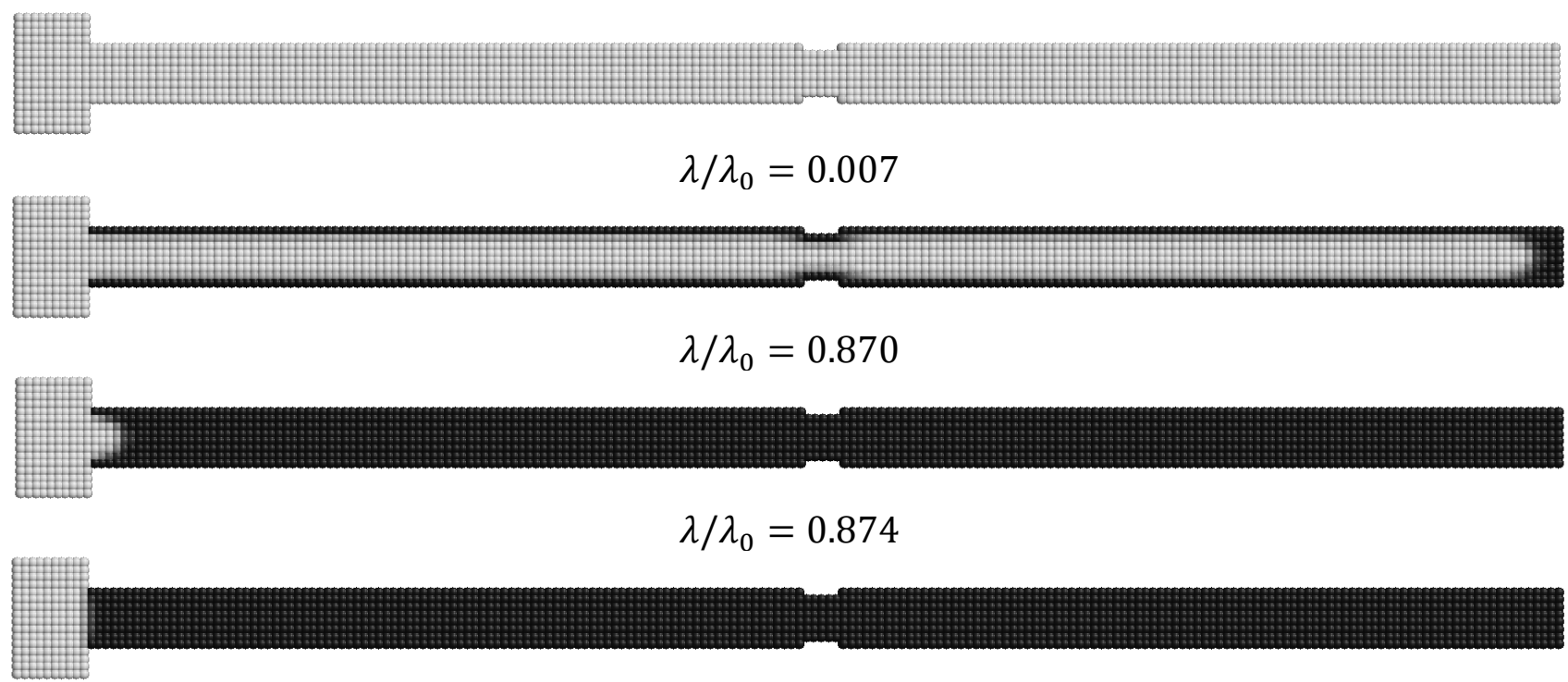

$$
\lambda / \lambda_{0}=1.000
$$




$$
\lambda / \lambda_{0}=0.861
$$

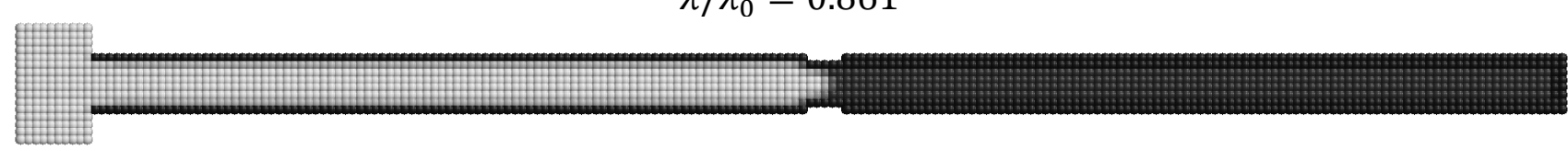

$$
\lambda / \lambda_{0}=0.799
$$

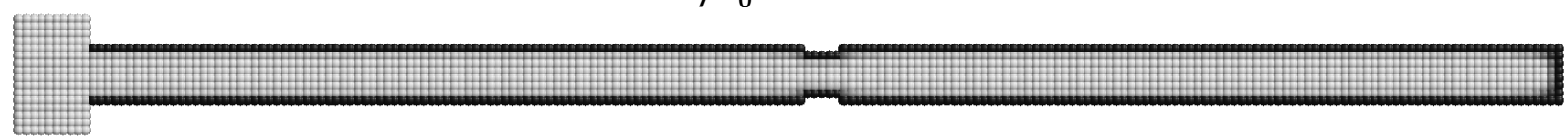

$$
\lambda / \lambda_{0}=0.795
$$

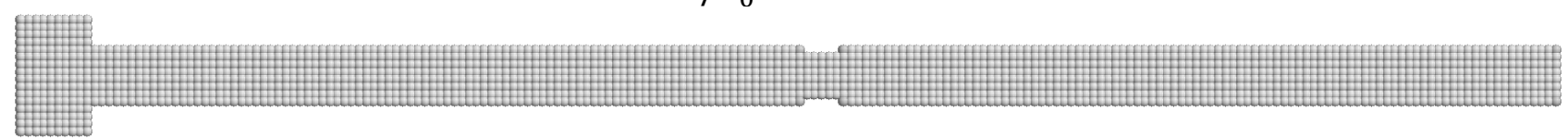

$$
\lambda / \lambda_{0}=0.007
$$

(b)

Figure S7. Visualizations of fluid density states at different relative activities $\left(\lambda / \lambda_{0}\right)$ in the close-ended lattice pore model of 200-sites length and 8-sites width without (a) and with (b) a pore narrowing of 6-sites width. The states in (a) are for adsorption and those in (b) are for adsorption and desorption. 